\title{
Disaster Planning During SARS-CoV-2/COVID: One Radiology Informatics Team's Story
}

\author{
Alexander J. Towbin ${ }^{1,2}$ (D) Jennifer Regan ${ }^{1}$ - David Hulefeld ${ }^{1}$ - Eric Schwieterman ${ }^{1} \cdot$ Laurie A. Perry $^{1} \cdot$ Sarah O$^{\prime}$ Brien $^{1}$. \\ Akhil Dhamija $^{1,3} \cdot$ Timothy OConnor $^{1} \cdot$ Jay A. Moskovitz ${ }^{1}$
}

Received: 24 June 2020 / Revised: 5 November 2020 / Accepted: 11 January 2021 / Published online: 18 February 2021

(c) Society for Imaging Informatics in Medicine 2021

\begin{abstract}
Disasters cause a major disruption to normal operations. Hospital information systems are often well-prepared for events such as fires or natural disasters. This type of disaster planning focuses on redundancy and manual workarounds. The SARS-CoV-2/COVID pandemic represented a new type of disaster for our radiology informatics team. In this pandemic, the information systems continued to work but the employees, and the computers that they worked with, had to be distanced. The purpose of this manuscript is to discuss the four phases of the disaster planning process: mitigation, planning, response, and recovery. We will illustrate the process with the example of how our radiology informatics team responded to the SARSCoV-2/COVID pandemic.
\end{abstract}

Keywords Downtime $\cdot$ Enterprise imaging $\cdot$ PACS $\cdot$ Disaster

\section{Introduction}

Disasters are unplanned events that cause a negative impact and disrupt a system's standard operations. These events place a significant strain on the human and physical resources of an organization and society [1]. The impact of this strain is often felt for months or years after the disaster event. While many disasters, such as fires and earthquakes, occur suddenly without warning, others, such as hurricanes or pandemics, may occur with variable lead time. Prior work has shown that organizations that prepare and plan for disasters fare better than those who do not [2].

In one sense, the move to digital operations in the early 2000s forced radiology departments to begin to plan for disasters [3-7]. Over the past two decades, most radiology departments have had to deal with unplanned downtimes of their critical systems, such as the picture archiving and

Alexander J. Towbin

alexander.towbin@cchmc.org

1 Cincinnati Children's Hospital, Department of Radiology, Cincinnati, $\mathrm{OH}$, USA

2 University of Cincinnati College of Medicine, Department of Radiology, Cincinnati, OH, USA

3 University of Toledo College of Medicine and Life Sciences, Toledo, OH, USA communication system (PACS). Because of the relative frequency of these downtimes, most radiology departments have documented downtime policies and procedures. These policies and procedures have variable levels of detail and are used to help guide radiology department staff when a system is not functioning to its desired state. Additionally, most hospitals have purchased and installed disaster recovery and business continuity solutions for their critical systems [8-11].

Unfortunately, unplanned system downtimes are a relatively frequent occurrence in many radiology departments. These unplanned downtimes are often the result of a small number of system issues and may cause or contribute to enterprise-wide issues. Thus, radiology department staff are adept at maintaining operations while their critical systems are not functioning, and information technology staff are skilled at fixing the underlying system issue. Because radiology staff are used to this type of unplanned downtime and have dealt with it frequently, we have anecdotally found that many departments have become complacent and have not planned for other downtime or disaster scenarios.

In our department, we realized that we had become complacent in our disaster preparedness. In 2010, we had replaced our first PACS and, with the replacement, we had installed a true business continuity system with duplicate copies of images. Over the next 8 years, we worked to refine 
our business continuity process and eliminate even planned downtime. This effort was successful, and we had operated for several years without an unplanned PACS downtime. That changed when a standard server operating system security patch was incompatible with the PACS application and caused both our production and business continuity environments to fail within minutes of each other. This led to an extended downtime of more than $10 \mathrm{~h}$. While we were able to manage the downtime, we realized that we had not planned for such a scenario and were thus unprepared in many ways. Over the ensuing 2 years, we have implemented a more formal disaster planning process and have solved many of the issues we faced on that day. Collaborative exercises, such as tabletop disaster scenarios, have helped us to identify holes in our disaster processes and address them in our policies and procedures. While we realize that we cannot predict every scenario, this more formal planning process has allowed us to be more prepared when a true disaster strikes. The purpose of this manuscript is to describe the phases of disaster response and how we have organized our radiology information technology disaster planning around these phases. Throughout this manuscript, we will share examples of how this planning influenced our preparations and response to the SARS-CoV-2/COVID pandemic. It does not encompass all of the work that occurred in the department related to our technical staff and patient care.

\section{Phases of a Disaster Response}

There are four phases of disaster response: mitigation, planning, response, and recovery [1]. These phases are often illustrated as a cycle (Fig. 1a) with one phase leading to the next. Even though this cycle is never-ending, the image of disaster response as a cycle is still not accurate as the different phases can occur simultaneously and out of sequence (Fig 1b). This has been illustrated throughout the SARSCoV-2/COVID pandemic. At the time of this writing (June

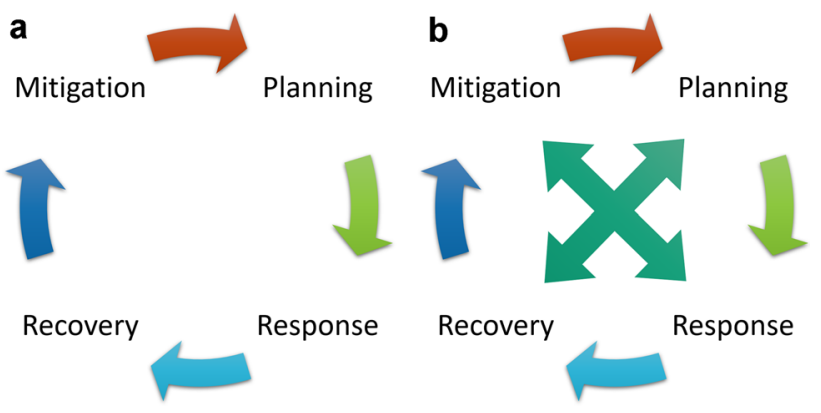

Fig. 1 a Graphic showing the typical representation of the disaster planning process as a cycle. b During the SARS-CoV-2/COVID pandemic, we have found the process to be less of a cycle but more of an interconnected web with each phase informing each of the other phases
2020), our hospital is currently in the early portions of the recovery phase of the SARS-CoV-2/COVID pandemic. Even though the hospital is working on recovery, we are also working to mitigate against further outbreaks, planning for the second wave of infection, if one occurs, and continuing to respond to the primary outbreak.

\section{Mitigation}

In the ideal state, most of the work related to disaster preparedness occurs in the mitigation and planning stages. The purpose of the mitigation phase is to implement systems or solutions to minimize the impact of a disaster [1]. Mitigation systems can include early warning or notification systems as well as solutions to address the disaster itself. For example, hospitals uniformly implement multiple solutions to mitigate against the impact of a fire. Smoke detectors and heat sensors couple with an alerting system to provide an early warning to hospital employees that a fire is present. Once a fire is detected, these early notification systems trigger multiple responses to minimize the danger and destruction of a fire. The triggered responses include sprinkler systems, door closures, evacuation orders, and notification of a local fire department.

As we began to understand the potential devastation of the SARS-CoV-2/COVID pandemic, we began our mitigation planning. In early February 2020, the radiology informatics team began discussing potential ways we could mitigate against the impact of SARS-CoV-2/COVID. Our mitigation strategy focused on social distancing. We knew that our highest risk areas were the radiologist reading rooms and the radiology department conference rooms.

We have multiple reading rooms in our department. Some specialty reading rooms are isolated and distinct, while others are pods, arising from a common reading room. The rooms are a mix of sizes and normally support 2-5 radiologists each. The informatics team determined two potential solutions to enable distancing of radiologists; they could either work from their home or their office. The team then worked to explore the impact, advantages, and disadvantages of each solution.

\section{Working From Home}

First, we explored having radiologists work from home. At the outset, we were concerned about system performance related to the network speeds through the hospital virtual private network (VPN), the ability of radiologists to add the system to their home, and the ability of the radiology informatics team to decommission the workstations from the reading room and deploy them to the radiologists. With regards to system performance, we were specifically concerned with two systems, the PACS (Merge Halo; IBM Watson Health Imaging; 
Cambridge, MA) and the voice-dictation system (PowerScribe 360; Nuance Communications; Burlington, MA). The PACS downloads images before allowing the user to view the study. This occurs in one of two methods. Most studies are cached to the disk-based on their presence on the reading worklist. While pre-caching may be slower over VPN through a home internet service provider, we believed that this solution would allow for adequate performance. However, we remained concerned about ad-hoc queries for studies not on the worklist. For those studies, the image download begins when the study is opened. The dictation software provided a different challenge. From prior experience, we knew that having the user's voice profile on the system's server added a delay to the signing process of each dictation. We knew that we could address this by copying the user's voice file to the PACS workstation and making a system configuration change. To test this solution, the radiologist informatics leader (AJT) brought a PACS workstation home and evaluated system performance. While performance was acceptable, we expected performance to degrade as the pandemic struck because we anticipated that there would be a spike in network traffic and VPN usage with more employees working from home. The radiology informatics team worked with the hospital network team to discuss options for dedicated VPN traffic or ensuring that the potential number of VPN connections and bandwidth could be increased.

The informatics team was also concerned about the different variables needed for home setup. First, we knew that radiologists would not know how to setup a PACS workstation and its diagnostic monitors. Thus, we created detailed how-to instructions for system setup and labeled workstations, cables, and monitors. This allowed us to promote social distancing for the radiology informatics team members rather than have the team members travel to private radiologist residences. The radiologist informatics leader tested these instructions when he brought the system to his house. Additionally, the informatics team knew that some radiologists would not be able to provide a wired connection to their router. Thus, the team researched and procured wireless adapters for the PACS workstations. The radiologist informatics leader evaluated system performance at his house with both wired and wireless connections. The radiologist informatics leader felt like there was a noticeable degradation in the performance of the PACS using the wireless adapter. However, even with performance degradation, the solution was felt to be feasible and sufficient to perform standard work tasks.

The final concern of the radiology informatics team related to system deployment. Our department was fortunate to have enough PACS workstations so that each faculty radiologist and fellow could take a machine home. However, this would leave less than five machines in the department. The team anticipated that it would take at least $30 \mathrm{~min}$ to decommission each machine. The decommissioning process would involve shutting down the computer, unplugging each input, labeling each connection and cable, and safely packaging the different components. We anticipated that this process would take 20-25 person-hours to complete but could be distributed amongst multiple members of the team. To prepare for this process, we procured bubble wrap and cardboard to protect the monitors and plastic bags to better transport the cables. Additionally, we discussed a schedule of how we could rapidly decommission the workstations.

\section{Working From Offices}

The plan of working from offices had several advantages, but also provided unique challenges. Our department is fortunate that each faculty radiologist has his or her own private office. The office spaces are isolated from the clinical space of the department and are large enough to be able to add a complete PACS setup. However, our 9 fellows work in a shared space that would not allow for appropriate distancing. Thus, we had to identify spaces for the fellows to work. We solved this by commandeering offices from our overnight and emeritus faculty, using several ancillary reading spaces that were traditionally used for research or training, and by using the office of a faculty member who had recently left the department.

We knew that the system performance would be better in offices on the hospital network as compared to home. However, each office already had a personal computer attached to the network. This would not be an issue for many radiologists as they could use the PACS workstation for other tasks such as email, internet usage, and word processing. However, this solution would not work for some radiologists as they would need to use their office computer for specific research tasks or other work. Thus, we asked each radiologist if he or she would need to use their office computer. If a radiologist needed to use his or her office computer, we worked with our hospital's network team to activate a second network jack in their office.

The final challenge of working from offices was similar to the challenge of working from home. The radiology informatics team had to decommission the machines from the reading room and transport them to the radiologist's office space. While this solution was also time-consuming, the team felt that it would take less time to complete as they would be moving the machines themselves. The challenge with this solution would be in rapidly moving a large number of machines across the street from our reading space to the office space. The informatics team researched options including renting a moving van. However, after discussion, the team determined that the move would be feasible using their cars on a weekend day. 


\section{Conference Room and Consultants in the Reading Room}

While we had options to allow radiologists to distance while performing their primary work, we realized that we had to provide a solution so that they could continue to provide their expertise to the numerous clinical consultants that stopped by our reading rooms each day. In addition to these clinical consults, the team had to provide a solution to allow our numerous daily conferences to proceed.

Fortunately, we already used a remote conferencing tool (Zoom Video Communications; San Jose, CA) to broadcast our conferences across our various locations. Our radiologists and clinical colleagues were familiar with this software. The informatics team knew that we would be able to continue to use the software throughout the SARS-CoV-2/ COVID pandemic. However, our conferences were run on a workstation in the conference room with specialized video outputs. We had to ensure that the system would work on our PACS workstations with multiple monitors in portrait orientation.

The radiology informatics team also believed that the conferencing software would be a useful tool to provide virtual consults. The team believed that the radiologist could initiate the conference on-demand, share his or her screen, and review an imaging study with the clinical team. The informatics team first tested the video conferencing software on the PACS workstation to understand how it would work with multiple monitors. The team determined that the entire PACS application was not viewable during a video conference. Instead, the radiologist could only display one monitor at a time. The informatics team used this information to create a how-to guide for radiologists.

Once the informatics team deemed that this type of virtual consult was feasible, we procured five additional software licenses to support the different clinical subspecialties. Each license was configured similarly so that radiologists could easily run a virtual consult for their service.

\section{Planning}

The disaster planning phase often relies on the creation of a written plan that details the steps to take during a disaster event. Our hospital and the radiology department have plans documented for different disasters such as fire, tornado, and an active shooter. The radiology informatics team has built detailed plans for managing system downtimes. In these system downtime plans, the informatics team has considered a range of scenarios such as when the electronic health record is offline, when the PACS is offline, and when the dictation system is offline. Each scenario has a different response detailed for each user type. We recently completed a disaster drill for a scenario where our production PACS and business continuity systems are both offline.

The SARS-CoV-2/COVID pandemic provided a unique challenge for the radiology informatics team as we had not previously planned for this scenario. Early scientific reports describing the experience in China highlighted the importance of chest radiography and chest CT in diagnosing an infection, especially when a polymerase chain reaction test was not available [12-14]. Based on this experience, we were anticipating having an influx of chest X-ray and chest CT studies.

The informatics team thought that the best way to manage these unique studies was to create a unique order within the electronic health record. This order would allow us to trigger unique imaging protocols and a unique structured report on the modalities and in the voice dictation system, respectively. As the team designed this order, we determined that it could be useful in future potential pandemics. Thus, we named the order using our standard order names for chest radiograph and chest $\mathrm{CT}$. However, for each order, we added the acronym PARC (pandemic acute respiratory contagion).

The radiologist informatics leader worked with the Thoracoabdominal section leader of our department to create and implement a unique CT protocol and to devise a unique standard structured report based on the findings described in the literature to that point [15-17]. It is important to note that the COVID-RADS system did not yet exist [18].

\section{Response}

The response phase of a disaster often gets the most attention because it is observed and judged by a larger group of people. The response phase can be subdivided into activation, notification, organization, implementation, and stabilization [1].

\section{Activation}

Activation occurs at the onset of a disaster and can be automated or manual. For example, when a fire occurs, the response can be activated either via automatic detection of smoke or a temperature change or via the manual trigger of a fire alarm. In a pandemic, the tools for activation are not as well defined. In our department, we did not implement our plans until our state's governor began to implement social distancing regulations. At this point, the Radiologist-in-Chief and radiologist informatics leader met and decided on the go-forward plan. They reviewed the informatics team's work and recommendations and decided that the ideal go-forward plan would be to close the reading room effective at the end of the day (a Friday) and move the PACS workstations to radiologist's offices over the weekend. However, because each radiologist had a different individual risk-profile, 
radiologists would be provided with the option to take their workstation home.

\section{Notification}

Notification occurs when the affected population is alerted that a disaster is in progress and that operations must be shifted to the disaster plan. The notification event is often tightly coupled to activation. For example, in a fire, the fire alarm goes off as soon as the smoke and/or heat sensor identifies a risk or as soon as the fire alarm is pulled.

In our department, once we decided to activate the disaster plan, we had to notify the radiologists and technologists of what our response would entail. To accomplish this, an emergency faculty meeting was called. At this meeting, the radiologist informatics leader discussed the plan and how it would be implemented. The radiologists were able to discuss the different options and ask questions of the leadership team. The departmental leadership asked the radiologist to respond within $3 \mathrm{~h}$ if they wanted to take their workstation home.

After the faculty meeting, the radiologist informatics leader sent an e-mail to all the department's employees notifying the staff of the changes to the radiologist staffing in the reading room as well as other changes to operations and patient care. This information was simultaneously added to the departmental Web site.

\section{Organization}

The organization phase typically represents a pause where the response team reviews their plan and the role each member will play in the response. During a fire, our hospital relies on captains for each area to ensure that the evacuation is orderly and complete.

During the pandemic, once the department was notified, the informatics team had to plan the move to the offices. We had already anticipated that a move would have to occur over the weekend and were prepared to implement the move the next day. The informatics team met after the emergent faculty meeting and reviewed the plan, identified the time that we would begin the move, and discussed what was needed to ensure success.

\section{Implementation}

During the implementation phase, the disaster plan is put in place and used to manage ongoing operations. Depending on the nature of the disaster, this phase may go exactly as planned, or the plan may need to be tweaked as the disaster persists and conditions change.

To implement our plan, the radiology informatics team had to move 30 of the department's 47 PACS workstations to radiologists' offices; four machines were sent to radiologists' homes. The remaining workstations either needed to remain in the reading room to allow for minimal radiologist presence or were located in one of our alternate reading spaces for residents and fellows.

The informatics team began decommissioning computers on Friday afternoon in preparation for a Saturday morning move. By the end of the day, Friday, approximately half of the computers that needed to be moved were decommissioned and placed on carts to be moved to the office space the next day. On Saturday morning, four members of the informatics team loaded the machines into their cars and moved them to the office space across the street. After the machines were unloaded, one member went back to the department and began decommissioning the remaining machines while the other three team members set up the machines in the office and tested their performance. The entire move took about $8 \mathrm{~h}$.

Typically, the radiology informatics team provides deskside support for the reading room and technologist quality control areas. Because they would be working from home for the foreseeable future, the team needed a way to continue to be able to provide support to the end-users. The team had experience with supporting applications while off-site through a remote support tool (BeyondTrust; Atlanta, GA). While software support was possible, hardware support would be difficult. The informatics team reviewed the logs from our online ticketing system and determined that peripheral devices such as microphones, keyboards, and mice were the most common cause of hardware failure. Baskets of these devices were placed in secure locations in the main reading room and at the radiologists' office space.

In the week following the move of PACS workstations, several other changes were implemented to enable social distancing and allow other groups (such as administrative assistants, schedule coordinators, coders, and 3D postprocessing technologists) to work from home. For the radiology informatics team, these changes required securing VPN access, enabling remote desktop functionality on certain work computers, forwarding work phones to personal cell phones, and creating how-to documents to teach users this new functionality.

\section{Stabilization}

Stabilization is typically the part of the response when the problem is corrected. The disaster plan has been implemented and tweaked and normal operations are proceeding along with the plan. Depending on the type of disaster, the stabilization phase may be the longest portion of a disaster response. During the SARS-CoV-2/COVID, this phase lasted for months.

Once a disaster plan has been implemented, success is often measured by the quality and frequency of communication 
from the team managing the disaster. Frequent communication allows the public to maintain a sense of control even as their normal world is in chaos. Additionally, if communication is allowed to be bidirectional, the response team can learn from the public's perception of the response or identify components of the response that are not working or were overlooked.

The radiology informatics team managed communication internally and externally through a series of daily huddles, frequent detailed emails to the department's staff, and frequent updates to the department's website. The daily huddles occurred via video conferencing software. The informatics team and its members managed huddles for three different groups, the radiologists, the technologist managers, and the informatics team itself. Being a part of each huddle allowed the team to provide updates and listen to feedback from each group. This feedback was used to modify the response plan. For example, as the daily volumes decreased, more radiologists were able to be moved from the reading room to different office spaces. The informatics team was able to accommodate these changes and move workstations as needed.

\section{Recovery}

The recovery phase occurs after the disaster has abated and the response team determines that the system can prepare to move back towards its baseline operations [1]. Unfortunately, during the SARS-CoV-2/COVID pandemic, there was no clear breakpoint to determine that we had entered the recovery phase. This breakdown in communication occurred because of mixed signals from the federal and state governments. In our hospital, plans for recovery began to take shape in mid-April and began to be implemented in mid-May.

The study volume in our department reached its nadir at approximately $40 \%$ of our normal volumes. We are currently performing at $88 \%$ of our normal volumes. As the volumes have increased, we have noticed a strain on our systems. This strain has been felt primarily by the faculty radiologists as they work in the reading room. Normally, when the reading rooms are fully staffed, the burden of phone calls and interruptions is distributed amongst multiple radiologists. While the number of interruptions has decreased, it is a greater burden to the radiologist working by him/herself in the room.

The SARS-CoV-2/COVID pandemic has also differed from other types of disasters in that the recovery is slow because the disease remains in the community. As we have eased into recovery, we have tried to balance the need to provide healthcare with the need to protect our patients and employees. For the Radiology Informatics team, the largest remaining task of recovery is related to moving the workstations back to the reading rooms. This move will be staged so that the reading rooms remain only partially staffed allowing the radiologists to remain distanced, while at the same time able to teach and provide direct oversite to the new fellowship class that will be starting in July.

In most disaster responses, the recovery phase ends with a debrief event. This event allows the response team to review their successes as well as identify areas for future improvement. Depending on the type of disaster, a debrief can include a formal investigation, a public forum for response, and/or an internal team meeting. Because the SARS-CoV-2/COVID is an ongoing pandemic, our team has not been able to have a complete debrief event. We have used our daily huddles to make ongoing adjustments and have incorporated what we have learned from the planning process in our recovery.

As the months have passed since start of the pandemic, we have continued to evaluate our department's response and the effect that the response has had on our ongoing operations. Over this time, we have had to make modifications to address new demands. For example, in March, we believed that the pediatric radiology fellows in our department could work in isolation, similar to the faculty. However, in July, the department believed that the training of our new fellows would suffer if the radiologists continued to work in isolation. This belief was couple with new evidence that mask wearing and social distancing could help to prevent transmission of the virus. Based on these two pieces of information, we planned and implemented a partial move back to the reading room. As we made this move the informatics team worked to maintain distancing in the reading rooms by limiting the number of workstations and maximizing distance between two workstations. The team also identified new spaces and converted them to reading rooms, making sure that network jacks were active, any windows had black-out curtains, and that desks and chairs were available. This partial move allowed most radiologists to move from working in their offices or at home to working from the reading room. Because we were not able to create enough space for all services to co-locate at least two radiologists (a faculty and a trainee), radiologists on some services continue to work from their office or from home. We believe that we will be able to move all radiologists back to the reading room once the pandemic is over.

While some changes are temporary, we believe that other changes will persist after the pandemic resolves. For example, all of our clinical conferences moved from being in-person to being virtual during the pandemic. We believe that all of our clinical conferences will continue to have a virtual component going forward. This change will persist due to providers increased comfort with the technology and our hospital's large physical footprint spanning multiple locations across our city. 


\section{Conclusion}

The SARS-CoV-2/COVID pandemic has been a unique disaster in the USA and the world. Our radiology informatics team has typically prepared for information systems disasters. This type of typical disaster preparedness focuses on redundancy and simplicity. While hardware can be involved in this type of disaster planning, it is focused on server and network infrastructure. The SARS-CoV-2/COVID forced our team to think differently about disaster planning and use new concepts such as social distancing and tools such as virtual conferencing to meet our department's needs. While we recognize that some of our success has been fortuitous (having enough PACS workstations for each radiologist and having enough office space), we believe that a large portion of our initial success was related to our detailed contingency planning process.

\section{Declarations}

\author{
Alex Towbin: \\ Advisory board: KLAS, IBM Watson Health imaging \\ Consultant: Applied Radiology \\ Author Royalties: Elsevier
}

Funding Grant Funding: Guerbet, Cystic Fibrosis Foundation.

\section{References}

1. Furin MA: Disaster Planning. https://emedicine.medscape.com/ article/765495-overview. Accessed June 19, 2020

2. Hugelius K, Becker J, Adolfsson A: Five Challenges When Managing Mass Casualty or Disaster Situations: A Review Study. Int J Environ Res Public Health 17(9), 2020 https://doi.org/10.3390/ ijerph17093068

3. Khorasani R: Business continuity and disaster recovery: PACS as a case example. J Am Coll Radiol: JACR 5 (2):144-145, 2008 https://doi.org/10.1016/j.jacr.2007.11.002

4. Khorasani R: What you can do to minimize system downtime. J Am Coll Radiol: JACR 4 (4):252-253, 2008 https://doi. org/10.1016/j.jacr.2007.01.005

5. Shapiro JS, Kannry J, Lipton M, Goldberg E, Conocenti P, Stuard S, Wyatt BM, Kuperman G: Approaches to patient health information exchange and their impact on emergency medicine. Ann Emerg Med 48(4):426-432, 2006 https://doi.org/10.1016/j. annemergmed.2006.03.032
6. Kroken P: Imaging center emergency: who has the back-ups? Radiol Manage 27(2):12-13, 2005

7. Wendt G, Peppler W, Edwards W: PACS disaster recovery procedures--evaluation in a clinical environment. J Digit Imaging 15 Suppl 1:112-113, 2002 https://doi.org/10.1007/s10278-002-5065-2

8. Becker M, Goldszal A, Detal J, Gronlund-Jacob J, Epstein R: Managing a Multisite Academic-Private Radiology Practice Reading Environment: Impact of IT Downtimes on Enterprise Efficiency. J Am Coll Radiol: JACR 12 (6):630-637, 2015 https:// doi.org/10.1016/j.jacr.2014.11.002

9. Berkowitz SJ, Wei JL, Halabi S: Migrating to the Modern PACS: Challenges and Opportunities. Radiographics: a review publication of the Radiological Society of North America, Inc 38(6):1761-1772, 2018 https://doi.org/10.1148/rg.2018180161

10. Kolowitz BJ, Lauro GR, Barkey C, Black H, Light K, Deible $\mathrm{C}$ : Workflow continuity--moving beyond business continuity in a multisite 24-7 healthcare organization. J Digit Imaging 25(6):744750, 2012 https://doi.org/10.1007/s10278-012-9504-4

11. Langer SG, Wood CP, Murthy NS, French TL, Rubin M: PACS bypass: a semi-automated routing solution to enable filmless operations when PACS fails. J Digit Imaging, 25(4):466-470, 2012 https://doi.org/10.1007/s10278-011-9446-2

12. Ai T, Yang Z, Hou H, Zhan C, Chen C, Lv W, Tao Q, Sun Z, Xia L: Correlation of Chest CT and RT-PCR Testing in Coronavirus Disease 2019 (COVID-19) in China: A Report of 1014 Cases. Radiology: 200642, 2020 https://doi.org/10.1148/radiol.2020200642

13. Fang Y, Zhang H, Xie J, Lin M, Ying L, Pang P, Ji W: Sensitivity of Chest CT for COVID-19: Comparison to RT-PCR. Radiology: 200432, 2020 https://doi.org/10.1148/radiol.2020200432

14. Xie X, Zhong Z, Zhao W, Zheng C, Wang F, Liu J: Chest CT for Typical 2019-nCoV Pneumonia: Relationship to Negative RTPCR Testing. Radiology: 200343, 2020 https://doi.org/10.1148/ radiol.2020200343

15. Bai HX, Hsieh B, Xiong Z, Halsey K, Choi JW, Tran TML, Pan I, Shi LB, Wang DC, Mei J, Jiang XL, Zeng QH, Egglin TK, Hu PF, Agarwal S, Xie F, Li S, Healey T, Atalay MK, Liao WH: Performance of radiologists in differentiating COVID-19 from viral pneumonia on chest CT. Radiology: 200823, 2020 https:// doi.org/10.1148/radiol.2020200823

16. Caruso D, Zerunian M, Polici M, Pucciarelli F, Polidori T, Rucci C, Guido G, Bracci B, de Dominicis C, Laghi A: Chest CT Features of COVID-19 in Rome, Italy. Radiology: 201237, 2020 https://doi. org/10.1148/radiol.2020201237

17. Wong HYF, Lam HYS, Fong AH, Leung ST, Chin TW, Lo CSY, Lui MM, Lee JCY, Chiu KW, Chung T, Lee EYP, Wan EYF, Hung FNI, Lam TPW, Kuo M, Ng MY: Frequency and Distribution of Chest Radiographic Findings in COVID-19 Positive Patients. Radiology: 201160, 2019 https://doi.org/10.1148/radiol. 2020201160

18. Salehi S, Abedi A, Balakrishnan S, Gholamrezanezhad A: Coronavirus disease 2019 (COVID-19) imaging reporting and data system (COVID-RADS) and common lexicon: a proposal based on the imaging data of 37 studies. Eur Radiol, $2020 \mathrm{https}: / /$ doi. org/10.1007/s00330-020-06863-0

Publisher's Note Springer Nature remains neutral with regard to jurisdictional claims in published maps and institutional affiliations. 